\title{
Acetazolamide attenuates cardiac fibrosis induced by aortic constriction through inhibiting transforming growth factor-\$1/Smad2 signaling pathway in mice
}

\author{
QIANQIAN HUO $^{1 *}$, TING WANG ${ }^{2 *}$, TAO WANG $^{3}$ and RUI ZHANG ${ }^{4}$ \\ ${ }^{1}$ Department of Cardiology, Jining No. 1 People's Hospital, Jining, Shandong 272011; \\ ${ }^{2}$ Department of Cardiology, Zoucheng People's Hospital, Zoucheng, Shandong 273500; \\ ${ }^{3}$ Department of Cardiology, Jiyang People's Hospital, Jiyang, Shandong 251400; \\ ${ }^{4}$ Department of Cardiology, Affiliated Hospital of Jining Medical University, \\ Jining, Shandong 272029, P.R. China
}

Received June 4, 2018; Accepted January 15, 2019

DOI: $10.3892 /$ etm.2019.7210

\begin{abstract}
The effect and mechanism of acetazolamide on cardiac fibrosis induced by transverse aortic constriction (TAC) were investigated. C57BL/6 mice were subjected to TAC or sham operation and then were orally gavaged with acetazolamide $(20 \mathrm{mg} / \mathrm{kg} /$ day $)$. After 4 weeks of operation, cardiac function was detected by echocardiography. Interstitial fibrosis was stained with Masson's trichrome. The expression of $\alpha$-smooth muscle actin ( $\alpha$-SMA), collagen I, transforming growth factor- $\beta 1$ (TGF- $\beta 1$ ) and Smad2 were measured by western blotting. The TAC mice displayed significant cardiac dysfunction and fibrosis. The expression of $\alpha$-SMA, collagen I, TGF- $\beta 1$ and $\mathrm{p}-\mathrm{Smad} 2$ in the TAC group was higher than those in the sham group. By contrast, acetazolamide administration inhibited interstitial fibrosis, as well as improved cardiac dysfunction induced by TAC. Acetazolamide also reduced the expression of $\alpha$-SMA, collagen I, TGF- $\beta 1$ and $p-S m a d 2$ in the TAC mice. Acetazolamide was able to attenuate cardiac fibrosis and improve cardiac dysfunction. The molecular mechanism involved in the anti-fibrotic effect of acetazolamide possibly was through inhibiting TGF- $\beta 1 / \mathrm{Smad} 2$ signaling pathway.
\end{abstract}

\section{Introduction}

Cardiac fibrosis is characterized by the excessive proliferation of interstitial fibroblasts and excessive deposition of extracellular

Correspondence to: Dr Rui Zhang, Department of Cardiology, Affiliated Hospital of Jining Medical University, 89 Guhuai Road, Rencheng, Jining, Shandong 272029, P.R. China

E-mail: hagxo908@163.com

${ }^{*}$ Contributed equally

Key words: cardiac fibrosis, pressure overload, acetazolamide, transforming growth factor- $\beta 1$ matrix. It is a common pathophysiologic mechanism during the development of various cardiovascular diseases such as atrial fibrillation, hypertensive heart disease, myocardial infarction and valvular heart diseases. Cardiac fibrosis has a key role in ventricular remodeling. The main pathological manifestations of cardiac fibrosis is myocardial stiffness increase, myocardial systolic and diastolic dysfunction, and eventually leading to heart failure and sudden death $(1,2)$. The current treatments available for cardiac fibrosis are not highly specific and often have many side effects. Therefore, a novel potential therapeutic agent for cardiac fibrosis is needed.

Acetazolamide is a carbonic anhydrase inhibitor, which is mainly applied for correct metabolic alkalosis (3) and edematous diseases such as COPD (4), cerebral edema (5) and chronic heart failure (6). Moreover, Li et al (7) also reported that acetazolamide could suppress tumor angiogenesis and metastasis in a Lewis lung carcinoma mouse model. Recently, Lin et al (8) reported that acetazolamide could enhance the cardioprotective effect of remifentanil in a rat model of myocardial ischemia/reperfusion injury. However, the effect of acetazolamide on cardiac fibrosis has not yet been confirmed. We hypothesized that acetazolamide may have potential usefulness in attenuating cardiac fibrosis. In this study, we created a mouse model of pressure overload induced by aortic constriction to investigate the effect of acetazolamide on cardiac fibrosis and the potential molecular mechanism.

\section{Materials and methods}

Reagents. Acetazolamide was purchased from Sigma-Aldrich (Merck KGaA, Darmstadt, Germany). The rabbit anti- $\alpha$-SMA, collagen I, TGF- $\beta 1$ and Smad 2 primary antibodies were purchased from Santa Cruz Biotechnology, Inc. (Dallas, TX, USA).

Ethics statement. Male C57BL/6 mice (8-10 weeks old) were provided by the Animal Experiment Center of Affiliated Hospital of Jining Medical University (Jining, China). All aspects of the experimental protocols were approved by the 

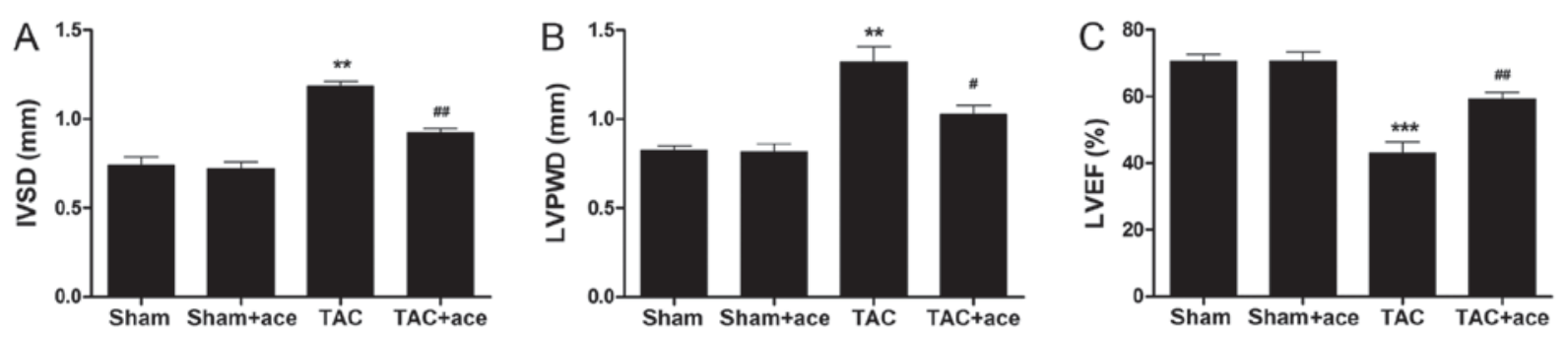

Figure 1. Cardiac function in each group. (A) IVSD in each group. (B) LVPWD in each group. (C) LVEF in each group. Ace, acetazolamide; IVSD, interventricular septum diastolic dimension; LVPWD, left ventricular posterior wall thickness diastole; LVEF, left ventricular ejection fraction. ${ }^{* *} \mathrm{P}<0.01$ and *** $\mathrm{P}<0.001$ vs. sham; ${ }^{*} \mathrm{P}<0.05$ and ${ }^{\# \#} \mathrm{P}<0.01$ vs. TAC.

Animal Care and Use Committee of Affiliated Hospital of Jining Medical University and conducted in accordance with the Guide for the Care and Use of Laboratory Animals, published by the US National Institutes of Health $(\mathrm{NIH}$ Publication no. 85-23, revised 1996). The mice were housed in a temperature controlled room $\left(21 \pm 2^{\circ} \mathrm{C}\right)$ with a relative humidity range of 30 to $40 \%$ on a 12:12-h light/dark cycle (lights on at 06:00). All rats had free access to water and food.

Animal model of pressure overload. The mice were anesthetized with an initial $4 \%$ isoflurane followed by a maintenance dose of $2 \%$ isoflurane, then intubated and ventilated. A midline incision was made at the sternum. After opening the mediastinal space, the aortic arch was blunt dissected at the base of the heart. A blunt 27-G injection needle (OD $0.4 \mathrm{~mm}$ ) was placed parallel to the aorta between the left carotid and the right innominate arteries, then the needle and the aortic arch were tied together using a 7-0 suture. After removing the needle, a model of aortic constriction was created. Sham mice underwent the same surgical procedure, the 7-0 suture was placed in the same position without ligation. After transverse aortic constriction (TAC) or sham operation, the mice were orally gavaged with acetazolamide $(20 \mathrm{mg} / \mathrm{kg} / \mathrm{day})$. There are four groups in this experiment: i) sham group; ii) sham+acetazolamide group; iii) TAC group; iv) TAC + acetazolamide group, $\mathrm{n}=10$ mice in each group. After 4 weeks of operation, all mice were sacrificed and the hearts were harvested. The heart samples were frozen in liquid nitrogen frozen and then stored at $-70^{\circ} \mathrm{C}$.

Echocardiography. After 4 weeks of operation, the mice were anesthetized by isoflurane and the cardiac function was detected using a rodent animal ultrasonic instrument (Vevo 2100; VisualSonics, Inc., Toronto, ON, Canada). The interventricular septum diameter (IVS), left ventricular (LV) posterior wall thickness (LVPW) and LV ejection fraction (LVEF) were calculated.

Western blotting. Total proteins were isolated from heart tissues using a protein extraction kit (Nanjing KeyGen Biotech Co., Ltd., Nanjing, China). Total protein concentration was calculated by bicinchoninic acid (BCA) Protein Assay Kit (Pierce, Rockford, IL, USA). Gel electrophoresis (10\%) was performed to separate the different molecular weight proteins and then transferred onto polyvinylidene difluoride membranes. A total of $30 \mu \mathrm{g}$ proteins were added into per lane for the electrophoresis. Bull Serum Albumin (BSA)
Blocking buffer (5\%) was used as the blocking reagent. The membrane was incubated with $\alpha$-SMA, collagen I, TGF- $\beta 1$, phospho-Smad 2 and Smad 2 for overnight at $4^{\circ} \mathrm{C}$. After incubation with the primary antibodies, the membrane was washed in Tris-buffered saline-tween (TBST) and then incubated with the HRP-conjugated secondary antibody at room temperature for another $2 \mathrm{~h}$. Rabbit polyclonal $\alpha$-SMA antibody (dilution, 1:1,000; cat. no. ab5694); rabbit monoclonal collagen I antibody (dilution, 1:1,000; cat. no. ab138492); rabbit monoclonal TGF- $\beta 1$ antibody (dilution, 1:1,000; cat. no. ab215715); rabbit monoclonal phospho-Smad2 antibody (dilution, 1:1,000; cat. no. ab188334); rabbit monoclonal Smad2 antibody (dilution, 1:1,000; cat. no. ab40855); rabbit polyclonal GAPDH antibody (dilution, 1:1,000; cat. no. ab37168) and secondary goat anti-rabbit (HRP) IgG antibody (dilution, 1:2,000; cat. no. ab6721) were all purchased from Abcam (Cambridge, MA, USA). Immuno-reactive bands were visualized by enhanced chemiluminescence (ECL) detection kit (Amersham Biosciences, Foster City, CA, USA). ImageJ software (NIH, Bethesda, MD, USA) was used to measure the blot signal and density.

Histological assessment of cardiac fibrosis. The LV tissue samples were fixed in paraformaldehyde $(3.7 \%$ in phosphate-buffered saline (PBS), freshly prepared) for $24 \mathrm{~h}$ and then embedded in paraffin. LV sections (4-5 $\mu \mathrm{m})$ were stained with Masson's trichrome for interstitial fibrosis. The proportion of the total fibrosis area was observed using a microscope (Nikon, Tokyo, Japan) and was calculated by ImageJ software (NIH), as the blue-stained areas divided by the total LV area.

Statistical analysis. SPSS 19.0 software (IBM Corp., Armonk, NY, USA) was used for statistical analysis. All results were presented as means \pm standard deviation (means $\pm \mathrm{SD}$ ). One-way ANOVA followed by post hoc test (Least Significant Difference) was used to compare the differences among the different groups. Student's t-test was used to compare the differences between the two groups. $\mathrm{P}<0.05$ was considered to indicate a statistically significant difference.

\section{Results}

Acetazolamide attenuates cardiac dysfunction and interstitial fibrosis induced by TAC. As shown in Fig. 1, the interventricular septum diastolic dimension (IVSD) and LV posterior wall thickness diastole (LVPWD) were significantly thicker in the TAC mice than those in the sham mice $(\mathrm{P}<0.01, \mathrm{P}<0.01$, 

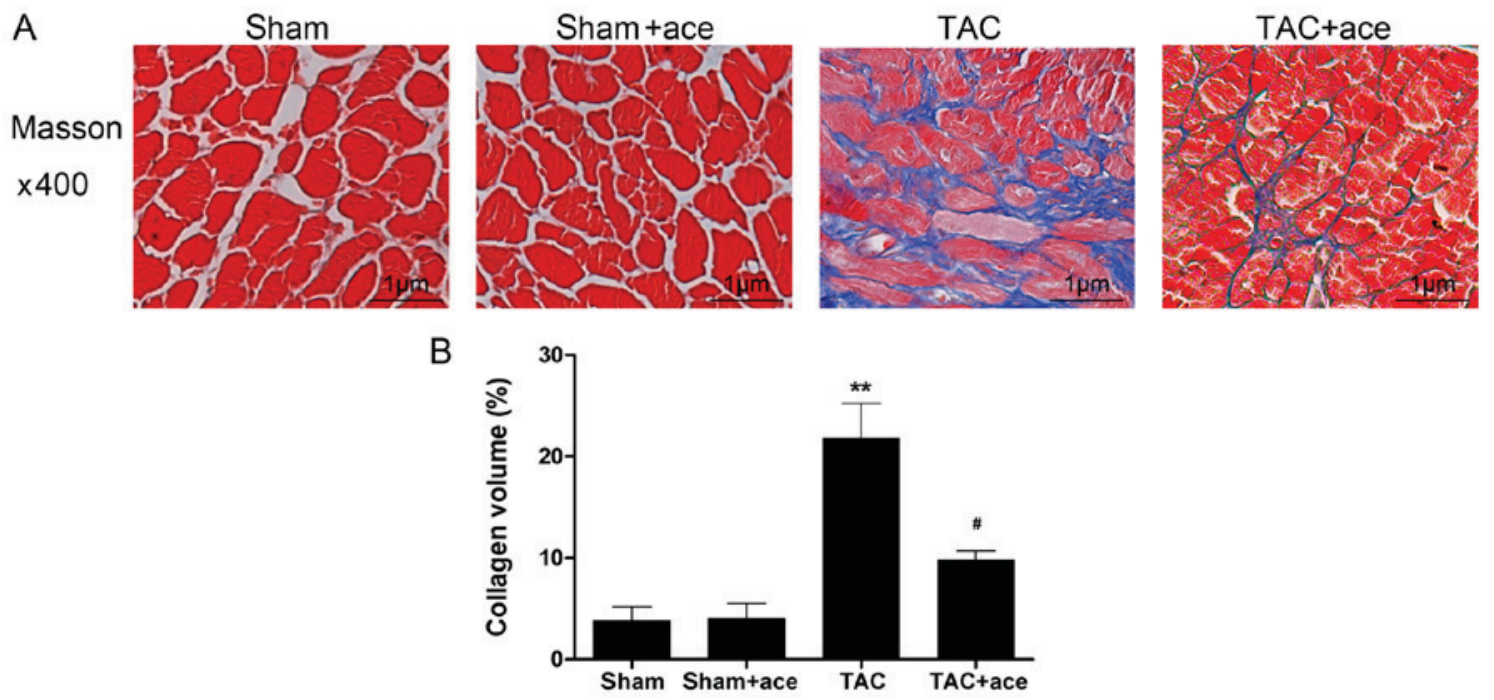

Figure 2. Assessment of myocardial fibrosis in each group. (A) Representative photographs of interstitial fibrosis stained by Masson's trichrome. (B) Collagen volume fraction. Ace, acetazolamide. ${ }^{* *} \mathrm{P}<0.01$ vs. sham; ${ }^{*} \mathrm{P}<0.05$ vs. TAC.

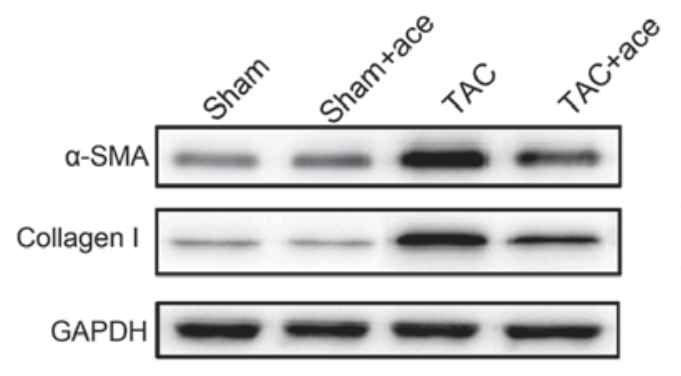

Figure 3. The expression of $\alpha$-SMA and collagen I proteins in each group. Ace, acetazolamide. ${ }^{* * *} \mathrm{P}<0.001$ vs. sham; ${ }^{\# \#} \mathrm{P}<0.01$ vs. TAC.
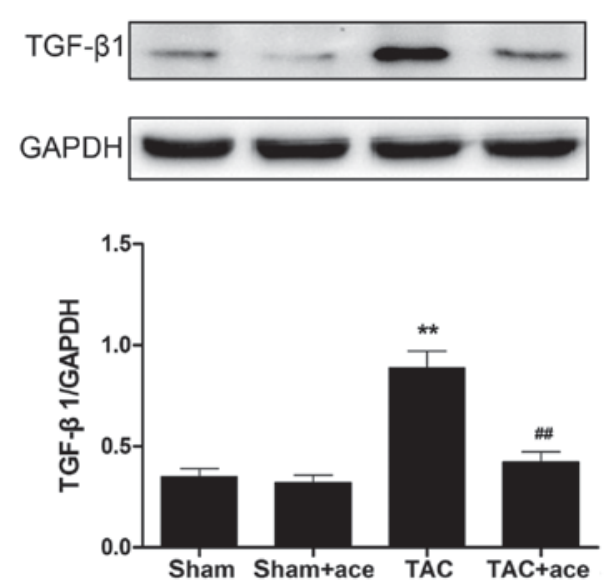
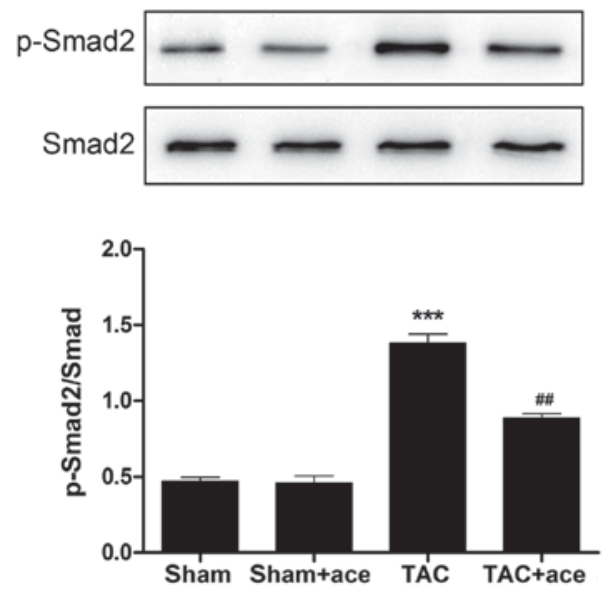

Figure 4. The expression of TGF- $\beta 1$, p-Smad2 and Smad2 proteins in each group. Ace, acetazolamide. ${ }^{* *} \mathrm{P}<0.01$ and ${ }^{* * *} \mathrm{P}<0.001$ vs. sham; ${ }^{\# \#} \mathrm{P}<0.01$ vs. TAC.

respectively). Moreover, the LVEF was significantly decreased in the TAC mice compared with the sham mice $(\mathrm{P}<0.001)$. By contrast, acetazolamide administration significantly decreased the IVSD and LVPWD, and inhibited the reduction in LVEF induced by $\mathrm{TAC}(\mathrm{P}<0.01, \mathrm{P}<0.05, \mathrm{P}<0.01$, respectively).

As shown in Fig. 2, the interstitial collagen volume was substantially increased in the TAC group compared with the sham group $(\mathrm{P}<0.01)$. By contrast, acetazolamide administration significantly inhibited TAC-induced interstitial fibrosis $(\mathrm{P}<0.05)$.

Acetazolamide inhibits the TAC-induced increase in the expression of $\alpha$-SMA and collagen I proteins. As shown in Fig. 3 , the expression of $\alpha$-SMA and collagen I proteins were significantly increased in TAC group compared with the sham group $(\mathrm{P}<0.001, \mathrm{P}<0.001$, respectively). Acetazolamide 
administration reduced the expression of $\alpha$-SMA and collagen I proteins in contrast to the TAC group $(\mathrm{P}<0.01$, $\mathrm{P}<0.01$, respectively).

Acetazolamide inhibits the activation of TGF- $\beta 1 /$ Smad 2 signaling pathway. As shown in Fig. 4, the expression of TGF- $\beta 1$ and phosphorylation level of Smad2 were significantly increased in TAC group compared with the sham group $(\mathrm{P}<0.01, \mathrm{P}<0.001$, respectively). Acetazolamide administration reduced the expression of TGF- $\beta 1$ and phosphorylation level of Smad2 in contrast to the TAC group $(\mathrm{P}<0.01, \mathrm{P}<0.01$, respectively).

\section{Discussion}

To the best of our knowledge, the present study provides the first report that acetazolamide is able to inhibit cardiac fibrosis and dysfunction induced by pressure overload in mice. The anti-fibrotic effect of acetazolamide was confirmed by the reduction of collagen volume in myocardial interstitium and the inhibition of $\alpha$-SMA and collagen I protein expression. Acetazolamide also inhibited the activation of TGF- $\beta 1 / \mathrm{Smad} 2$ signaling pathway. These findings support the conclusion that acetazolamide possibly is a potential therapeutic agent for the prevention of cardiac fibrosis.

Cardiacfibrosisisanimportanthallmark during thedevelopment of ventricular remodeling, and is a key pathological foundation of cardiac dysfunction and malignant cardiovascular events (2). The development of cardiac fibrosis is associated with the activation of renin angiotensin-aldosterone system, oxidative stress and a variety of cytokines. TGF- $\beta 1$ is a cytokine that performed a variety of biological functions including promoting cell proliferation and differentiation, promoting collagen synthesis, and inhibiting collagen degradation. It has been demonstrated that TGF- $\beta 1$ is one of the most important factors for inducing cardiac fibrosis (9). During the development of cardiac fibrosis, activated TGF- $\beta 1$ can promote cardiac fibroblast differentiation into myofibroblasts, as reflected by the expression of $\alpha$-SMA (10). Rosenkranz et al (11) reported that the overexpression of TGF- $\beta 1$ could induce cardiac fibrosis and hypertrophy in transgenic mice. Furthermore, in a rat model of pressureoverload, Kuwahara et al (12) found that TGF- $\beta 1$ function blocking could inhibit cardiac fibrosis and dysfunction. Collagen I is secreted by myofibroblasts and is the most abundant collagen type in the myocardium, constituting $~ 80 \%$ of the extracellular matrix (1). The overexpression of collagen I in transgenic mice displayed significant cardiac fibrosis and dysfunction (13). Similarly, in this study, we created a pressure overload model to induce cardiac fibrosis. Our results showed that the mice displayed significant cardiac fibrosis after 4 weeks of TAC, as confirmed by Masson staining and increased collagen volume. The expression of TGF- $\beta 1$, $\alpha$-SMA and collagen I proteins was also markedly increased in the pressure-overloaded myocardium. Acetazolamide administration significantly attenuated cardiac fibrosis and inhibited the expression of TGF- $\beta 1, \alpha$-SMA and collagen I in the pressure-overloaded myocardium.

TGF- $\beta 1 /$ Smad signaling is the main pathway during the development of cardiac fibrosis $(9,14)$. The protein Smads are the key downstream signaling molecules triggered by TGF- $\beta 1$ and then induce the expression of pro-fibrotic target genes (9). Lei et al (15) reported that Smad2 siRNA could significantly inhibit TGF- $\beta 1$-induced fibrotic changes in rat cardiac fibroblasts. Huang et al (16) also found that Smad3 activation could induce cardiac fibrosis in a myocardial remodeling model. Conversely, Smad7 activation could inhibit cardiac fibrosis and dysfunction induced by angiotensin II (17). Similarly, in this study, we found that the phosphorylation level of Smad2 was significantly increased in the TAC mice. Acetazolamide administration reduced the phosphorylation level of Smad2 in the pressure-overloaded myocardium. Our results demonstrated that acetazolamide significantly attenuated cardiac fibrosis and dysfunction induced by pressure overload through inhibiting the TGF- $\beta 1 / \mathrm{Smad} 2$ signaling pathway.

In conclusion, this is the first study to identify that acetazolamide inhibit the development of cardiac fibrosis. The molecular mechanism involved in the anti-fibrotic effect of acetazolamide was possibly through inhibiting TGF- $\beta 1 /$ Smad 2 signaling pathway. Our results suggest that acetazolamide may be used as a therapeutic agent for the prevention of cardiac fibrosis. Further research is needed to investigate the effect and mechanism of acetazolamide in cardiac fibroblasts in vitro.

\section{Acknowledgements}

Not applicable.

\section{Funding}

No funding was received.

\section{Availability of data and materials}

All data generated or analyzed during this study are included in this published article.

\section{Authors' contributions}

QH and RZ designed the study and performed the experiments. QH, TiW and TaW established the animal models. QH and TiW collected the data. TiW and TaW analyzed the data. $\mathrm{QH}$ and RZ prepared the manuscript. All authors read and approved the final manuscript.

\section{Ethics approval and consent to participate}

This study was approved by the Animal Care and Use Committee of Affiliated Hospital of Jining Medical University (Jining, China).

\section{Patient consent for publication}

Not applicable.

\section{Competing interests}

The authors declare that they have no competing interests. 


\section{References}

1. Creemers EE and Pinto YM: Molecular mechanisms that control interstitial fibrosis in the pressure-overloaded heart. Cardiovasc Res 89: 265-272, 2011.

2. Kong P, Christia P and Frangogiannis NG: The pathogenesis of cardiac fibrosis. Cell Mol Life Sci 71: 549-574, 2014.

3. Bar A, Cies J, Stapleton K, Tauber D, Chopra A and Shore PM: Acetazolamide therapy for metabolic alkalosis in critically ill pediatric patients. Pediatr Crit Care Med 16: e34-e40, 2015.

4. Fontana V, Santinelli S, Internullo M, Marinelli P, Sardo L, Alessandrini G, Borgognoni L, Ferrazza AM, Bonini M and Palange P: Effect of acetazolamide on post-NIV metabolic alkalosis in acute exacerbated COPD patients. Eur Rev Med Pharmacol Sci 20: 37-43, 2016.

5. Bremer AM, Yamada K and West CR: Ischemic cerebral edema in primates: Effects of acetazolamide, phenytoin, sorbitol, dexamethasone, and methylprednisolone on brain water and electrolytes. Neurosurgery 6: 149-154, 1980.

6. Apostolo A, Agostoni P, Contini M, Antonioli L and Swenson ER: Acetazolamide and inhaled carbon dioxide reduce periodic breathing during exercise in patients with chronic heart failure. J Card Fail 20: 278-288, 2014.

7. Li XJ, Xiang Y, Ma B and Qi XQ: Effects of acetazolamide combined with or without $\mathrm{NaHCO}_{3}$ on suppressing neoplasm growth, metastasis and aquaporin-1 (AQP1) protein expression. Int J Mol Sci 8: 229-240, 2007.

8. Lin PT, Chen WH, Zheng H, Lai ZM and Zhang LC: Involvement of AQP 1 in the cardio-protective effect of remifentanil post-conditioning in ischemia/reperfusion rats. Int J Clin Exp Med 8: 12736-12745, 2015.

9. Dobaczewski M, Chen W and Frangogiannis NG: Transforming growth factor (TGF) $-\beta$ signaling in cardiac remodeling. J Mol Cell Cardiol 51: 600-606, 2011.

10. Wu M, Han M, Li J, Xu X, Li T, Que L, Ha T, Li C, Chen Q and Li Y: 17beta-estradiol inhibits angiotensin II-induced cardiac myofibroblast differentiation. Eur J Pharmacol 616: 155-159, 2009.
11. Rosenkranz S, Flesch M, Amann K, Haeuseler C, Kilter H, Seeland U, Schlüter KD and Böhm M: Alterations of betaadrenergic signaling and cardiac hypertrophy in transgenic mice overexpressing TGF-beta(1). Am J Physiol Heart Circ Physiol 283: H1253-H1262, 2002.

12. Kuwahara F, Kai H, Tokuda K, Kai M, Takeshita A, Egashira K and Imaizumi T: Transforming growth factor-beta function blocking prevents myocardial fibrosis and diastolic dysfunction in pressure-overloaded rats. Circulation 106: 130-135, 2002.

13. Miller AD and Tyagi SC: Mutation in collagen gene induces cardiomyopathy in transgenic mice. J Cell Biochem 85: 259-267, 2002.

14. Bujak M and Frangogiannis NG: The role of TGF-beta signaling in myocardial infarction and cardiac remodeling. Cardiovasc Res 74: 184-195, 2007.

15. Lei B, Hitomi H, Mori T, Nagai Y, Deguchi K, Mori H, Masaki T, Nakano D, Kobori H, Kitaura Y, et al: Effect of efonidipine on TGF- 31 -induced cardiac fibrosis through Smad2-dependent pathway in rat cardiac fibroblasts. J Pharmacol Sci 117: 98-105, 2011.

16. Huang XR, Chung AC, Yang F, Yue W, Deng C, Lau CP, Tse HF and Lan HY: Smad3 mediates cardiac inflammation and fibrosis in angiotensin II-induced hypertensive cardiac remodeling. Hypertension 55: 1165-1171, 2010.

17. Wei LH, Huang XR, Zhang Y, Li YQ, Chen HY, Yan BP, Yu CM and Lan HY: Smad7 inhibits angiotensin II-induced hypertensive cardiac remodelling. Cardiovasc Res 99: 665-673, 2013.

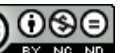

This work is licensed under a Creative Commons Attribution-NonCommercial-NoDerivatives 4.0 International (CC BY-NC-ND 4.0) License. 